\title{
Nivel de ansiedad de los pacientes antes de ingresar a la consulta odontológica
}

\section{Dental Anxiety Before a Dentist Appointment}

\section{Nível de ansiedade em pacientes antes de ingressar à consulta odontológica}

\author{
Marta Inés Ferreira-Gaona, DDS ${ }^{1}$; \\ Clarisse Virginia Díaz-Reissner, MSc²*; \\ Nohelia María Pérez-Bejarano, DDS³; \\ Natalia Cristina Cueto-González, Univ.4; \\ Tatiana Guadalupe Leggio-González, Univ.4; \\ Laura Beatriz Cardozo-Lovera, Univ.4; \\ Gabriela Belén Jiménez-Ariste, Univ.4; \\ Álvaro Vidal González-Zarza, Univ.4; \\ Fabiola Matiauda-Lazzeri, Univ.4; \\ Ana Elizabeth Ovelar-Benítez, Univ. ; \\ Zoraida Beatriz Aguilera-Peralta, Univ. ${ }^{4}$
}

Recibido: 28 de agosto de 2017 / Aceptado: 14 de mayo de 2018

Dor: http://dx.doi.org/10.12804/revistas.urosario.edu.co/revsalud/a.7266

Para citar este artículo: Ferreira-Gaona MI, Díaz-Reissner CV, Pérez-Bejarano NM, Cueto-González NC, Leggio-González TG,

Cardozo-Lovera LB, et al. Nivel de ansiedad de los pacientes antes de ingresar a la consulta odontológica. Rev Cienc Salud.

2018;16(3):463-72. DoI: http://dx.doi.org/10.12804/revistas.urosario.edu.co/revsalud/a.7266

\section{Resumen}

Introducción: la ansiedad dental o miedo al tratamiento odontológico es una de las dificultades más frecuentes para el profesional, y esto puede tener un impacto considerable en la salud oral. El objetivo es evaluar el nivel de ansiedad de los pacientes que acudieron a las consultas en la clínica odontológica

1 Dirección de Investigación, Facultad de Odontología, Universidad del Pacífico, Paraguay.

2 Cátedra de Metodología de la Investigación y Bioestadística. Facultad de Odontología, Universidad del Pacífico, Paraguay.

Autora de correspondencia: diazclarisse@gmail.com

3 Cátedra de Metodología de la Investigación y Bioestadística. Facultad de Odontología, Universidad del Pacífico, Paraguay.

4 Carrera de Odontología, Universidad del Pacífico, Paraguay. 
de una empresa privada de servicios odontológicos, en 2015. Materiales y métodos: estudio transversal en el que se utilizó la Escala de Ansiedad Dental de Corah Modificada (mDAs), y se interrogó sobre la ansiedad que generan los componentes de la consulta odontológica. Resultados: participaron un total de 297 adultos de los cuales $54.85 \%$ fueron varones y la mediana de edad fue 28 años. Se obtuvo un bajo número de pacientes con ansiedad extrema (7.74\%), siendo el porcentaje más elevado para la inyección de anestésico local (85.52\%). El $17.85 \%$ obtuvo un nivel de ansiedad elevada y el $17.85 \%$ severa. El componente de la consulta odontológica que generó mayor nivel de ansiedad fue la extracción dental con $40.40 \%$. Conclusión: el paciente debe sentirse lo más relajado posible al ir a una consulta odontológica por lo que se necesitan charlas a los proveedores de salud dental acerca de las diferentes técnicas para disminuir la ansiedad dental del paciente.

Palabras clave: ansiedad al tratamiento odontológico, investigación conductual, escala de ansiedad manifiesta.

\section{Abstract}

Introduction: Dental anxiety or consulting fear to dentist appointment and/or dental treatment, is one of the most frequent problems to the professional which can have an impact to the oral health. The objective is to assess the level of anxiety in patients that attend to appointments in a dental clinic from a private company of dental care in the year of 2015. Materials and methods: The study design is cross-sectional, Corah's Modified Dental Anxiety Scale (MDAs) was utilized. And question were made about the components of dental services that generate dental anxiety. Results: The sample consisted of 297 adult patients, $54.85 \%$ were males and middle age was 28. It was obtained a low number of patients with extreme anxiety ( $7.74 \%$ ) according to the questions; being the highest percentage for local anesthetic injection for dental treatment (85.52\%). A $17.85 \%$ had a high level of anxiety and $17.85 \%$ had a severe level of anxiety. The component that caused anxiety the most was tooth extraction with a $40.40 \%$. Conclusion: The patient must feel as relax as possible when going to a dentist appointment that is why would be needed to speak with care providers about different techniques to reduce dental anxiety on patients.

Keywords: Dental anxiety, behavioral research, manifest anxiety scale.

\section{Resumo}

Introdução: a ansiedade dental ou medo ao tratamento odontológico, é uma das dificuldades mais frequentes para o profissional, que pode ter impacto na saúde oral. O objetivo é avaliar o nível de ansiedade dos pacientes que acudiram às consultas na clínica odontológica de uma empresa privada de serviços odontológicos, no ano 2015. Materiais e métodos: estudo transversal no qual utilizou-se a Escala de Ansiedade Dental de Corah Modificada (MDAs), e se interrogou sobre a ansiedade que geram os componentes da consulta odontológica. Resultados: participaram um total de 297 adultos dos quais $54.85 \%$ foram homens e a idade média foi 28 anos. Obteve-se um número baixo de pacientes com ansiedade extrema (7.74\%), senda a porcentagem mais elevado para a injeção de anestésico local (85.52\%). $017.85 \%$ obteve um nível de ansiedade elevada e o $17.85 \%$ severa. O componente da consulta odontológica que gerou maior nível de ansiedade foi a extração dental com $40.40 \%$. Conclusão: o paciente deve se sentir o mais relaxado possível ao ir a uma consulta odontológica pelo que precisam-se conversas aos provedores de saúde dental acerca das diferentes técnicas para diminuir a ansiedade dental do paciente.

Palavras-chave: ansiedade ao tratamento odontológico, pesquisa comportamental, escala de ansiedade manifesta. 


\section{Introducción}

T a ansiedad es una reacción biopsicológica frente a la vida (1). Constituye en principio una Urespuesta psicológica, anticipada a problemas reales o imaginarios, y que se perciben de acuerdo con las personas y su percepción de los problemas (2).

Es un fenómeno que se caracteriza por sentimientos subjetivos de tensión, aprensión, nerviosismo y preocupación experimentado por un individuo en un momento particular (3).

Por su parte, la ansiedad dental se ha descrito como el miedo a la consulta o al tratamiento odontológico, constituyéndose en una de las dificultades más frecuentes para el profesional, con gran impacto en la salud oral $(4,5)$.

Una mala experiencia durante un tratamiento dental pasado puede ser suficiente para que el paciente evite asistir al dentista. Aunque lo considere necesario, lo hará únicamente frente a la situación extrema que no le deje alternativa, dando como resultado una alteración de la conducta del paciente al asistir al consultorio odontológico, que resulta en la poca colaboración de este (6). El temor puede acrecentarse al punto de abandonar todo tratamiento, sea este leve o complicado $(7,8)$.

La ansiedad se considera una amenaza para muchos profesionales de la salud, la cual no tiene una relación específica en cuanto a género, y que cada individuo tiene un nivel diferente de ansiedad. El miedo es una respuesta biológica que presenta el individuo al encontrarse frente a una situación que considera amenazante $(9,10)$.

En la clínica, el paciente trae consigo una historia que refleja su propia percepción del entorno y la cavidad bucal es "su boca", la zona que, en la consulta, es la expresión de sus rasgos personales (11). En la consulta odontológica se invade ese espacio íntimo; por ello, es importante establecer una buena relación con el paciente y así ganar su confianza y que ello haga más agradable y, por tanto, más productiva la consulta, sin despertar temor a lo que vendría después (tratamiento) $(12,13)$.

Como se había mencionado, un paciente ansioso dificulta el tratamiento, pues suele amplificar tanto síntomas como sensaciones de dolor, no coopera y, como resultado, ambos (paciente y profesional) terminan estresados e insatisfechos, por lo cual se posterga el restablecimiento de la salud bucal (14).

El nivel de la ansiedad dental en los pacientes que acuden a la clínica odontológica se puede medir por medio de cuestionarios. Para el presente estudio esta se midió por medio de un cuestionario validado: Escala Corah de Ansiedad Dental Modificado (15). Este fue desarrollado en 1969, y una validación en español fue utilizada en un estudio de ansiedad del tratamiento estomatológico, la cual sirvió de base para este estudio $(16,17)$.

La empresa de servicios odontológicos Odontos de Promedent forma parte de un grupo empresarial ligado a los servicios de salud odontológica desde hace 3 lustros y presta 
consulta en varias especialidades a un aproximado de 13000 pacientes por mes, en sus más de 17 sucursales situadas en el casco urbano de distintas ciudades (18).

El objetivo del estudio fue describir el nivel de ansiedad de los pacientes que acuden a las consultas en la clínica odontológica en 2015.

\section{Materiales y métodos}

Elad

diseño de estudio aplicado fue transversal, para lo que se preparó un instrumento ajustado al cuestionario de Escala de Ansiedad Dental de Corah Modificada (MDAs) y con ello evaluar el componente de nivel de ansiedad $(15,16)$. En la primera parte de este estudio se consignaron los datos personales, la segunda parte correspondió a la medición de niveles de ansiedad, que incluían los procedimientos odontológicos y el nivel de ansiedad que particularmente podrían provocar. El protocolo de investigación fue aprobado por el Comité de Ética en Investigación de la Universidad del Pacífico.

La población de estudio quedó conformada por pacientes mayores de 18 años que acudieron a las clínicas odontológicas Odontos de Promedent, en 2015, y que aceptaron participar del estudio. Fueron consideradas las clínicas odontológicas de mayor afluencia, ubicadas en las ciudades de Asunción, Luque, Lambaré y San Lorenzo.

Para el cálculo del tamaño de la muestra, sobre una población estimada de 13000 pacientes que consultan mensualmente en las clínicas odontológicas Odontos de Promedent, con un nivel de confianza del $95 \%$, una precisión de $5 \%$ y una proporción esperada del 77.4\%, se necesitó reclutar 267 sujetos y, estimando una pérdida de la muestra del 10\%, se llegó a un tamaño muestral de 296 personas aplicando un muestreo no probabilístico de casos consecutivos $(15,18)$. El cálculo del tamaño muestral se realizó utilizando la calculadora del tamaño muestral diseñado en el software Excel (19).

Se reclutó a los pacientes en la sala de espera de la clínica, donde el encuestador explicó los objetivos del estudio, posteriormente se firmó el consentimiento informado y, por último, se aplicó el cuestionario. Una vez completada la encuesta, se les agradeció por su participación entregándoles un folleto con algunas estrategias de control de la ansiedad para uso personal.

Se aplicó estadística descriptiva utilizando frecuencia absoluta y porcentaje representados en tablas. Así también, se aplicó estadística inferencial para estimar el intervalo de confianza del nivel de ansiedad y la prueba Ji al cuadrado de Pearson de homogeneidad para comparar la distribución del nivel de ansiedad por sexo. Se utilizó un nivel de confianza del $95 \%$. Para el análisis de los datos se cargaron en una base diseñada en el software Excel de Microsoft Office versión 2010 y se analizaron con EpiInfo ${ }^{\mathrm{TM}}$ 7.1.4, desarrollado por el Center for Control Desease and Prevention (CDC) (20). 


\section{Resultados}

T a muestra quedó conformada por 297 pacientes adultos que acudieron a las distintas ـclínicas del seguro odontológico privado. El 54.85\% fue de sexo masculino y el $45.42 \%$ de sexo femenino. La mediana de edad fue de 28 años, siendo la edad mínima de 18 y la máxima de 73 años, el $25 \%$ de la muestra tenía menos de 23 años y el $75 \%$ menos de 40. En cuanto al nivel de escolaridad: el 54.88\% había finalizado la universidad, el $40.40 \%$ la secundaria, el $3.70 \%$ la primaria y el $1.01 \%$ no tenía estudios.

Se obtuvo en todas las preguntas un bajo número de pacientes con ansiedad extrema. La inyección de anestésico local para el tratamiento dental fue el que generó mayor ansiedad, considerando los niveles de leve a extremo, fue del 85.52\% (tabla 1).

Tabla 1. Distribución de respuestas al MDAS

\begin{tabular}{|c|c|c|c|c|c|}
\hline \multirow[b]{2}{*}{ Preguntas } & \multicolumn{5}{|c|}{ Sentimiento de ansiedad o preocupación } \\
\hline & 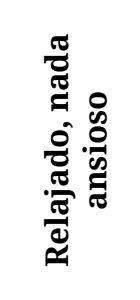 & 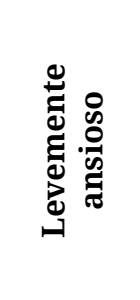 & 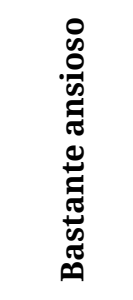 & 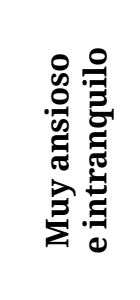 & 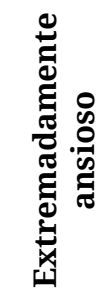 \\
\hline $\begin{array}{l}\text { - Si tuviera que ir al dentista ma- } \\
\text { ñana para una revisión, ¿cómo } \\
\text { se sentiría al respecto? }\end{array}$ & $45.79 \%$ & $37.04 \%$ & $11.45 \%$ & $4.71 \%$ & $1.01 \%$ \\
\hline \multirow{2}{*}{$\begin{array}{l}\text { - Cuando está esperando su turno } \\
\text { en el consultorio, ¿cómo se siente? }\end{array}$} & & & & & \\
\hline & $41.08 \%$ & $38.05 \%$ & $14.81 \%$ & $4.71 \%$ & $1.35 \%$ \\
\hline \multirow{2}{*}{$\begin{array}{l}\text { Cuando está en la silla del den- } \\
\text { tista esperando mientras él pre- } \\
\text { para el torno para comenzar de } \\
\text { trabajo en sus dientes, ¿cómo se } \\
\text { siente? }\end{array}$} & & & & & \\
\hline & $29.29 \%$ & $35.69 \%$ & $24.24 \%$ & $8.75 \%$ & $2.02 \%$ \\
\hline \multirow{2}{*}{$\begin{array}{l}\text { - Imagínese que está en la silla del } \\
\text { dentista para una limpieza. El } \\
\text { dentista saca los instrumentos } \\
\text { para raspar y pulir sus dientes, } \\
\text { ¿cómo se siente? }\end{array}$} & & & & & \\
\hline & $26.60 \%$ & $34.68 \%$ & $23.23 \%$ & $13.13 \%$ & $2.36 \%$ \\
\hline \multirow{2}{*}{$\begin{array}{l}\text { - Si le van a inyectar con una aguja } \\
\text { anestésico local para su trata- } \\
\text { miento dental ¿cómo se siente? }\end{array}$} & & & & & \\
\hline & $14.48 \%$ & $28.62 \%$ & $31.65 \%$ & $17.51 \%$ & $7.74 \%$ \\
\hline
\end{tabular}

Pacientes que acudieron al seguro odontológico privado. Paraguay, 2015 ( $n$ = 297). 
El 30.30\% presentó un nivel de ansiedad leve y el 39.06\% presentó un nivel de ansiedad moderado (tabla 2).

Tabla 2. Distribución del nivel de ansiedad

\begin{tabular}{lccccc} 
Nivel de ansiedad & No. & $\%$ & Acum. \% & $\begin{array}{c}\text { Lim. Inf. } \\
\text { (IC 95\%) }\end{array}$ & $\begin{array}{c}\text { Lim. Sup. } \\
\text { (IC 95\%) }\end{array}$ \\
\hline Leve & 90 & 30.30 & 30.30 & 25.13 & 35.88 \\
\hline Moderado & 116 & 39.06 & 69.36 & 33.47 & 44.86 \\
\hline Elevado & 38 & 12.79 & 82.15 & 9.22 & 17.14 \\
\hline Severo & 53 & 17.85 & 100.00 & 13.66 & 22.68 \\
\hline
\end{tabular}

Pacientes que acudieron al seguro odontológico privado. Paraguay, 2015 ( $n=297$ ).

El porcentaje más alto de ansiedad fue para el componente "que le tengan que extraer un diente” y el mayor porcentaje que no generó ningún sentimiento de ansiedad fue "olor a remedios de dentista” (tabla 3 ).

Tabla 3. Componentes de la consulta odontológica

\begin{tabular}{|c|c|c|c|c|c|}
\hline \multirow[b]{2}{*}{ Componentes } & \multicolumn{5}{|c|}{ Sentimiento de ansiedad o preocupación } \\
\hline & $\begin{array}{l}\text { Ninguna } \\
\%\end{array}$ & $\begin{array}{c}\text { Baja } \\
\%\end{array}$ & $\begin{array}{c}\text { Moderada } \\
\%\end{array}$ & $\begin{array}{c}\text { Alta } \\
\%\end{array}$ & $\begin{array}{c}\text { No sabe } \\
\%\end{array}$ \\
\hline $\begin{array}{l}\text { El sonido del torno o aparato } \\
\text { de limpieza }\end{array}$ & 20.88 & 34.68 & 33.00 & 10.77 & 0.67 \\
\hline $\begin{array}{l}\text { Saber que le tienen que } \\
\text { anestesiar para tratarlo }\end{array}$ & 17.51 & 33.00 & 32.32 & 15.82 & 1.35 \\
\hline $\begin{array}{l}\text { La sensación de } \\
\text { adormecimiento por anestesia }\end{array}$ & 23.23 & 33.00 & 31.31 & 11.11 & 1.35 \\
\hline $\begin{array}{l}\text { Pensar en abrir la boca } \\
\text { por mucho tiempo }\end{array}$ & 29.29 & 28.96 & 30.98 & 9.43 & 1.35 \\
\hline $\begin{array}{l}\text { No saber qué realmente le van } \\
\text { a hacer }\end{array}$ & 28.62 & 27.27 & 28.62 & 13.47 & 2.02 \\
\hline $\begin{array}{l}\text { Que le tengan que sacar el } \\
\text { diente }\end{array}$ & 10.10 & 19.53 & 28.28 & 40.40 & 1.68 \\
\hline $\begin{array}{l}\text { El olor a dentista o remedios } \\
\text { de dentista }\end{array}$ & 41.08 & 22.56 & 24.58 & 10.10 & 1.68 \\
\hline $\begin{array}{l}\text { Que el tratamiento sea } \\
\text { demasiado largo }\end{array}$ & 25.93 & 30.30 & 25.59 & 16.50 & 1.68 \\
\hline $\begin{array}{l}\text { Me da vergüenza cómo está mi } \\
\text { boca ahora }\end{array}$ & 26.26 & 26.26 & 29.97 & 16.50 & 1.01 \\
\hline
\end{tabular}

Pacientes que acudieron al seguro odontológico privado. Paraguay, 2015 ( $n=297$ ). 
Los niveles de ansiedad severo, elevado y moderado fueron ligeramente superiores para el sexo femenino, mientras que el nivel leve fue ligeramente superior para el sexo masculino (tabla 4), pero estas diferencias no resultaron estadísticamente significativas.

Tabla 4. Relación entre el nivel de ansiedad por sexo

\begin{tabular}{|c|c|c|c|c|c|}
\hline \multirow{2}{*}{ Sexo } & \multicolumn{5}{|c|}{ Nivel de ansiedad } \\
\hline & Severo & Elevado & Moderado & Leve & Total \\
\hline \multirow{2}{*}{$\begin{array}{l}\text { Femenino } \\
(n=146)\end{array}$} & & & & & \\
\hline & $19.86 \%$ & $13.01 \%$ & $43.15 \%$ & $23.97 \%$ & $100.00 \%$ \\
\hline \multirow{2}{*}{$\begin{array}{l}\text { Masculino } \\
(n=151)\end{array}$} & & & & & \\
\hline & $15.89 \%$ & $12.58 \%$ & $35.10 \%$ & $36.42 \%$ & $100.00 \%$ \\
\hline \multirow{2}{*}{$\begin{array}{c}\text { Total } \\
(n=297)\end{array}$} & & & & & \\
\hline & $17.85 \%$ & $12.79 \%$ & $39.06 \%$ & $30.30 \%$ & $100.00 \%$ \\
\hline
\end{tabular}

\section{Discusión}

l determinar el nivel de ansiedad de pacientes que acuden a la consulta dental en la clíInica de una empresa privada, e identificar los eventos que fueron más incidentes en los pacientes con alto nivel de ansiedad ante la consulta odontológica; se obtuvo como resultado un nivel de ansiedad dental moderado, teniendo como principal detonante la inyección del anestésico local.

Se debe considerar qué eventos traumáticos, en algún momento de la vida del paciente relacionados con la consulta dental, pudieron haber influenciado con respecto a los niveles de ansiedad en una consulta odontológica; existe una gran probabilidad de que el paciente abandone el tratamiento odontológico sin importar si es leve o complicado; las condiciones sociales y educativas podrían ser significativas si se evaluara la relación de esta con la ansiedad de cada paciente (21).

Diversos estudios encontraron significativamente mayores niveles de ansiedad dental en mujeres que en hombres (22-25). Sin embrago, en el estudio de Kirova y colegas, no se encontraron diferencias significativas entre la ansiedad y el sexo (26). Similar al resultado obtenido en este estudio, donde la diferencia de ansiedad fue ligeramente mayor en mujeres, pero no fue significativa.

En el estudio de Prathima y colegas, los mayores niveles de ansiedad se encontraron en pacientes debido a la "difícil experiencia previa en tratamientos dentales", seguido de "el 
taladro" y en tercer lugar “inyección” (27). En otro estudio realizado por Sghaireen y colegas, la inyección de la anestesia local fue el procedimiento dental más temido, en coincidencia con este estudio, generó nivel de ansiedad alto la "inyección de la anestesia local” y tener que "extraer un diente" (23). Popularmente, el sonido del torno se considera uno de los detonantes principales, en el estudio se observó que dos tercios de los encuestados reportaron niveles bajos y moderados de ansiedad, en tanto la quinta parte menciona que ese sonido no le genera ansiedad alguna.

Los profesionales necesitan ser educados sobre las causas de la ansiedad dental y recibir formación sobre cómo tratar el problema. Los altos niveles de ansiedad dental, según lo indicado por las cuentas del MDAs, se correlacionaron con las visitas dentales menos frecuentes, así como con ninguna visita a una clínica dental en los últimos años (28). Estas variables no fueron tenidas en cuenta en este estudio, por lo que se considera pertinente recomendar su importancia en un diseño adecuado; igualmente, se debería tener presente el registro del motivo de consulta. La ansiedad dental de los pacientes con la intervención temprana debe ser combinada con diferentes características de la edad, unas de las técnicas más utilizadas en el tratamiento de los trastornos de ansiedad es la desensibilización sistemática (29).

Se concluye que prevaleció un nivel moderado de ansiedad, aunque no alcanzó a ser mayoría; se encontró un bajo número de pacientes con ansiedad extrema y los que la presentaron, en general, refirieron la inyección de anestésico local para el tratamiento dental como el detonante principal.

\section{Descargos de responsabilidad}

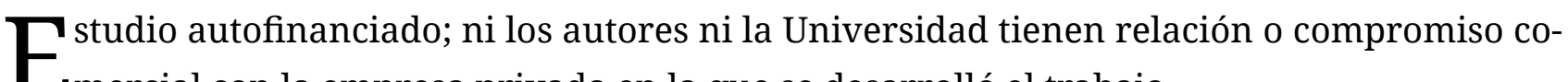
工mercial con la empresa privada en la que se desarrolló el trabajo.

\section{Referencias}

1. Trickett S. Supera la ansiedad y la depresión. Barcelona: Hispano Europea; 2012.

2. Caycedo C, Cortés OF, Gama R, Rodríguez H, Colorado P, Caycedo M, et al. Ansiedad al tratamiento odontológico: Características y diferencias de género. Suma Psicológica. 2008;15(1):259-78.

3. Krahn N, García A, Gómez L, Astié F. Fobia al tratamiento odontológico y su relación con ansiedad y depresión. Fundamentos en Humanidades. 2011;12(23):213-22. 
4. Bermudez-Cuesta MV, Diaz-Romero RM, LittmanSevilla J, Santos-Sotres J, Perez-Romero E, Ocaña-López F. Efectos de la musicoterapia sobre la ansiedad generada durante la atención dental en las mujeres embarazadas en el Servicio de Estomatología del Instituto Nacional de Perinatología. Rev ADM. 2014;LXI(2):59-64.

5. Díaz-Orueta U, Banterla F, Climent G. Isla Calma: realidad virtual para la distracción del dolor y la ansiedad en el afrontamiento del miedo al dentista. Ansiedad y Estrés. 2014;20(2/3):127-41.

6. Lima-Álvarez M, Casanova-Rivero Y. Miedo, ansiedad y fobia al tratamiento estomatológico. Rev Hum Med. 2006;6(1).

7. Oliva Te-kloot J. Bioethics and pain in dentistry: a humanistic approach. Rev Latinoam Bioet. 2015;15(1):38-53.

8. Marya CM, Grover S, Jnaneshwar A, Pruthi N. Dental anxiety among patients visiting a dental institute in Faridabad, India. West Indian Med J. 2012;61(2):187-90.

9. Sierra JC, Ortega V, Zubeidat I. Ansiedad, angustia y estrés: tres conceptos a diferenciar. Rev Mal-Estar Subj. 2003;3(1):10-59.

10. Rojas-Alcayaga G, Misrachi Launert C. La interacción paciente-dentista, a partir del significado psicológico de la boca. Av En Odontoestomatol. 2004;20(4):185-91.

11. Küstner EC, López JL. Esquemas de medicina bucal. Barcelona: Edicions Universitat Barcelona; 2010.

12. Mento C, Gitto L, Liotta M, Muscatello MRA, Bruno A, Settineri S. Dental anxiety in relation to aggressive characteristics of patients. Int J Psychol Res. 2014;7(2):29-37. DOI: $10.21500 / 20112084.656$

13. Sáenz SJ. Clínica de Odontología: la orientación hacia el paciente. Dent Clin Orientat Patient Engl. 2014;8(2):22-4.

14. Lima-Álvarez M, Rivero-Varona T, Pérez-Cederrón R. Factores de riesgo y fenómenos psicológicos relacionados con el estado de salud bucal. Rev Hum Med [internet]. 2007 [citado 2015 oct 27];7(3). Disponible en: http://bvs.sld.cu/revistas/revistahm/numeros/2007/ n21/body/hmc060307.htm

15. Mohammed RB, Lalithamma T, Varma DM, Sudhakar KNV, Srinivas B, Krishnamraju PV, et al. Prevalence of dental anxiety and its relation to age and gender in coastal Andhra (Visakhapatnam) population, India. J Nat Sci Biol Med. 2014;5(2):409-14. Dor: 10.4103/0976-9668.136210

16. Humphris G, Crawford JR, Hill K, Gilbert A, Freeman R. UK population norms for the modified dental anxiety scale with percentile calculator: adult dental health survey 2009 results. BMC Oral Health. 2013;13(1). Dor: 10.1186/1472-6831-13-29

17. Rodríguez-Chala HE, Marrero-Hernández M, Pazos-Rodríguez AR, Solar-Carballo O, CruzGarcía M, Méndez-Romero M, et al. Ansiedad al tratamiento estomatológico. Revista Médica Electrónica PortalesMedicos.com [internet]. 2014 [citado 2015 oct 27]; Ix(4). Disponible en: www.revista-portalesmedicos.com/revista-medica/ansiedad-al-tratamiento-estomatologico/

18. Odontos. Servicio Odontológico Prepago [internet]. 2015 [citado 2015 oct 27]. Disponible en: http://www.odontos.com.py/nosotros.php 
19. Pita-Fernández S. Determinación del tamaño muestral. Cad Aten Primaria. 1996;3:138-44.

20. Centers for Disease Control and Prevention. Epi Info TM 7 [internet]. 2015 [citado 2015 oct 27]. Disponible en: http://wwwn.cdc.gov/epiinfo/7/

21. Ríos-Erazo M, Herrera-Ronda A, Rojas-Alcayaga G. Ansiedad dental: evaluación y tratamiento. Av En Odontoestomatol. 2014;30(1):39-46. Dor: 10.4321/S0213-12852014000100005

22. Egbor PE, Akpata O. An evaluation of the sociodemographic determinants of dental anxiety in patients scheduled for intra-alveolar extraction. Libyan J Med. 2014;9:25433. Dor: 10.3402/ljm.v9.25433

23. Sghaireen MG, Zwiri AMA, Alzoubi IA, Qodceih SM, Al-Omiri MK. Anxiety due to dental treatment and procedures among university students and its correlation with their gender and field of study. Int J Dent. 2013;2013:5;647436. Dor: http://dx.doi. org/10.1155/2013/647436

24. de Carvalho RWF, Falcão PG, Campos GJ, Bastos A de S, Pereira JC, Pereira MA, et al. Anxiety regarding dental treatment: prevalence and predictors among Brazilians. Ciênc Amp Saúde Coletiva. 2012;17(7):1915-22.

25. Al-Omari WM, Al-Omiri MK. Dental anxiety among university students and its correlation with their field of study. J Appl Oral Sci. 2009;199-203. Dor: 10.1590/S167877572009000300013

26. Kirova DG, Atanasov DT, Lalabonova CK, Janevska S. Dental anxiety in adults in Bulgaria. Folia Med (Plovdiv). 2010;52(2):49-56. Dor: 10.2478/v10153-010-0047-5

27. Prathima V, Anjum MS, Reddy PP, Jayakumar A, Mounica M. Assessment of anxiety related to dental treatments among patients attending dental clinics and hospitals in Ranga Reddy District, Andhra Pradesh, India. Oral Health Prev Dent. 2014;12(4):357-64.

28. Levin L, Proter NE, Levin S. Dental visits and personality traits among young adults. Quintessence Int Berl Ger 1985. 2007;38(7):e379-83.

29. Luo H, Feng Y, Xu X, Zhang J. Correlation between anxiety, depression and pefectionistic tendencies in patients before orthodontic treatment. Shanghai Kou Qiang Yi Xue. 2014;23(5):609-13. 